\title{
Visceral pain triggered by traction on the ileocecal ligament with ileitis
}

\author{
Habibullah Janyaro* \\ Juan Wan* \\ Adnan H Tahir \\ Manoj K Shah \\ Xiao-Jing Li \\ Ming-Xing Ding
}

College of Veterinary Medicine, Huazhong Agricultural University,

Wuhan, People's Republic of China

*These authors contributed equally to this work.
Correspondence: Ming-Xing Ding College of Veterinary Medicine, Huazhong Agricultural University, I Shizishan Street, Hongshan District, Wuhan, Hubei 430070, People's Republic of China

Tel +86 I3808697687

Email dmx@mail.hzau.edu.cn
This article was published in the following Dove Press journal: Journal of Pain Research

30 September 2016

Number of times this article has been viewed

Background: Visceral pain is a common symptom of several gastrointestinal disorders. Despite tremendous progress in understanding its basic mechanisms, it remains a significant health challenge for clinicians. The present study quantified the intensity of visceral pain using ileocecal ligament traction on an inflamed ileum in goats.

Materials and methods: A total of 36 male goats weighing $20.05 \pm 2.1 \mathrm{~kg}$ were randomly allocated equally into a 2,4,6-trinitrobenzenesulfonic acid (TNBS) group $(n=18)$ and a saline group ( $\mathrm{n}=18$ ). Ileitis was induced via the injection of $30 \mathrm{mg}$ TNBS dissolved in $30 \%$ ethanol into the ileal wall through a laparotomy. An equal volume of normal saline was injected into the ileal wall of the saline goats. Behavioral responses to traction $(2,4$, and $6 \mathrm{~N})$ on the ileocecal ligament were observed on days 3, 7, and 14. Six goats from each group received a laparotomy and partial intestinal resection for ileal sample collection immediately after behavioral testing on days 3,7 , and 14 . Ileal histopathological changes were assessed and concentrations of myeloperoxidase, IL-1 $\beta$, IL-6, and TNF $\alpha$ investigated using enzyme-linked immunosorbent assay.

Results: The TNBS-treated goats exhibited remarkably increased macroscopic scores, mast-cell counts, myeloperoxidase, and TNF $\alpha$ concentrations on days 3 and 7 compared to the saline group, and increased microscopic scores and IL-1 $\beta$ and IL-6 concentrations on days 3-14. The TNBS-treated goats exhibited behavioral changes in response to traction in the same pattern as their microscopic changes and cytokine levels. The traction force correlated positively with pain-behavior responses. Conclusion: Traction on the ileocecal ligament of goats with ileitis provoked an apparent, stable, and reproducible ileum-derived pain. The current model may be helpful in evaluating the efficacy of new drugs for the management of visceral pain and in investigating its underlying mechanisms.

Keywords: goats, ileitis, 2,4,6-trinitrobenzenesulfonic acid, traction, cytokines

\section{Introduction}

Visceral pain is a complex and common symptom in clinical practice that occurs as a result of gastrointestinal surgery or disorders, such as obstruction, intussusceptions, neoplasms, volvulus, ischemia, infarction, luminal compression, intra-abdominal abscess, intestinal foreign body, celiac, typhlitis, intestinal or biliary colic, incarcerated or strangulated hernia, and intestinal and mesentery infarct. ${ }^{1-3}$ It may be accompanied by weight loss, bloating, diarrhea, diaphoresis, or any combination thereof, with changes in vital signs and emotional manifestations. Visceral pain is considered vague and often difficult to localize, and is more unpleasant than apparent intensity-matched somatic pain. ${ }^{4,5}$ Visceral pain produces a considerable negative impact on daily lives, causes a huge economic burden, and creates tremendous pressure on health care systems 
worldwide. ${ }^{6,7}$ The small intestine is more prone to intestinal disorders and pain than other segments of the gut, because of its greater length and enhanced peristaltic movement. The small intestine is the focus of common clinical presentations, and visceral pain remains a difficult entity to treat, because its etiopathogenesis is not completely understood. An animal model is needed to study the underlying pain mechanism and evaluate the efficacy of analgesics for the treatment of visceral pain originating from the small intestine.

Chemical and mechanical stimuli are commonly used to establish visceral pain models. Previous studies injected acetic acid into the peritoneal cavity to induce visceral pain and observed stretching behaviors (eg, contraction of the abdominal muscles, stretching of hind limbs, or rotation of the trunk) to assess pain. ${ }^{8,9}$ Visceral and somatic responses are evoked, because the peritoneum is partially a somatic structure. Therefore, the use of intraperitoneal irritants (eg, chemical stimuli) is nonspecific for the study of visceral pain. Most researchers have used colorectal distension to evoke pain responses in 2,4,6-trinitrobenzenesulfonic acid (TNBS)induced colitis. ${ }^{10-12}$ This method is used to measure visceral hyperalgesia. Several researchers used a traction method to assess mechanical pain responses in animal models. HolzerPetsche and Brodacz ${ }^{13}$ compared visceral pain responses to a noxious mechanical stimulus to those obtained with a chemical stimulus in anesthetized rats using graded traction (2-30 g) on a bundle of mesentery vessels. Ovarian ligament traction was used to study visceral pain in dogs. ${ }^{14}$ However, these experimental approaches used traction-evoked visceral pain from the mesentery and ovarian ligaments, but not smallintestine pain, which is most commonly seen in practice. These studies were also conducted for a short period (1-5 hours) in anesthetized animals, which may be inappropriate for prolonged durations that hinder the evaluation of the efficacy of long-acting analgesics.

Goats are medium-size docile animals that are convenient for the manipulation of traction-induced pain and observations of visceral pain responses. The present study established small-intestine pain caused by traction. TNBS was administered to induce ileitis and sensitize the pain response. Ileocecal ligament traction was used to evoke visceral pain. Behavioral change scores were used to evaluate visceral pain intensity.

\section{Materials and methods}

\section{Experimental animals and grouping}

All goats were thoroughly examined to ascertain their health status. A total of 36 apparently healthy hybrid male goats with an average age of $12 \pm 2.2$ months and an average weight of $20 \pm 2.1 \mathrm{~kg}$ were used for this study. Goats were purchased from the Hubei Agricultural Academy of Science and allotted into two groups: the TNBS group $(n=18)$ and the saline group $(n=18)$. Goats were dewormed and acclimated for 2 weeks prior to the commencement of experiments. Goats were maintained on daily rations, and water was available ad libitum. Special attention was paid to maintaining all goats under the same management conditions. The experiments were performed in a quiet environment, and the ambient temperature fluctuated from $22^{\circ} \mathrm{C}$ to $26^{\circ} \mathrm{C}$. All animal procedures used in this study were approved by the animal experimentation ethics committee of Huazhong Agricultural University (Wuhan, People's Republic of China [PRC], permit HZAUSH-2015-005), and were performed in accordance with the guidelines of the International Association for the Study of Pain.

\section{Induction of experimental ileitis}

Goats were fasted for 24 hours with free access to drinking water prior to ileal treatment with TNBS or saline. This fasting was necessary to avoid regurgitation and respiration complications during surgical procedures. Baseline cardinal parameters, such as pulse rate, respiratory rate, and body temperature, were recorded. Animals were premedicated with atropine $(0.02 \mathrm{mg} / \mathrm{kg}$ intramuscularly [IM]), etamsylate $(0.03 \mathrm{~g} / \mathrm{kg} \mathrm{IM})$, and xylazole $(0.10 \mathrm{mg} / \mathrm{kg} \mathrm{IM}$; North China Pharmaceutical Co Ltd, Shijiazhuang, PRC). Goats were placed in left lateral recumbency. Anesthesia was induced via an intravenous administration of ketamine $\mathrm{HCl}(0.5 \mathrm{mg} / \mathrm{kg} /$ min; YaoPharma Co Ltd, Chongqing, PRC). The anesthetic infusion was discontinued when the goats exhibited eyelidreflex loss, obtunded corneal reflexes, and no response to pricking of the legs or coronary hooves. The trachea was intubated to avoid aspiration of ruminal contents. Lidocaine (2\%; Shandong Hualu Pharmaceutical Co Ltd, Liaocheng, PRC) was used via infiltration. An intravenous infusion of ketamine during the surgery $(0.05 \mathrm{mg} / \mathrm{kg} / \mathrm{min})$ was initiated when the goats moved or struggled, and was continued until they reentered the anesthetic state. The abdomen was opened via a $6 \mathrm{~cm}$ incision in the right flank using aseptic surgical procedures to locate the ileum, and its terminal portion was exteriorized gently on moist sterile gauze. For the TNBS group, $1.2 \mathrm{~mL}$ of a solution of $30 \mathrm{mg}$ TNBS (Sigma-Aldrich, St Louis, MO, USA) dissolved in 30\% ethanol was injected into the ileal wall at five points, $\sim 15 \mathrm{~cm}$ proximal to the ileocecal junction, using a 30-gauge needle. The dose of TNBS was determined according to our pretest, in which 
this dose induced apparent inflammation in the ileum that was characterized by mucosal ulceration, wall thickness, and increased neutrophils and mast cells at the macroscopic or microscopic level. The saline group received the same volume of normal saline $(0.9 \% \mathrm{NaCl})$ injected in the same manner. Two fine-silk ligatures were loosely tied in the ileal mesentery just cranially and caudally to the injection sites for later location identification in both groups. A nylon thread (2-0) passed through a soft silicone tube ( $2 \mathrm{~mm}$ in diameter) was tied loosely to the ileocecal ligament near the injected segments of the ileum. The other end of the thread and tube was exteriorized through an Eppendorf tube (with its bottom removed), which was buried in the abdominal wall $3 \mathrm{~cm}$ caudal to the dorsal end of the original skin incision, and fixed inside the Eppendorf tube. The intestine was returned to the abdominal cavity, and the fixed thread on the ileocecal ligament was pulled using a dynamometer (device for measuring force; Figure 1) at forces of 2, 4, and $6 \mathrm{~N}$ before closing the abdominal wall. This procedure did not damage any visceral organ. The incision was closed routinely.

The goats were housed individually in a quiet environment and monitored until full recovery from the anesthesia. The goats received tramadol hydrochloride $(5 \mathrm{mg} / \mathrm{kg} \mathrm{IM}$; Hubei Qianjiang Pharmaceutical Co Ltd, Qianjiang, PRC) for 2 days for postoperative pain management. The goats recovered from the anesthesia without complications, and exhibited apparently normal behavior on the same day after surgery. Water was freely available 4 hours after the surgery, but grass was withheld until the day after surgery. The postoperative condition of the goats was monitored at least twice daily, and their wounds were treated with $1 \%$ povidone iodine solution daily until completely healed. Animals were weighed prior to the administration of TNBS or saline (day 0 ) and on days 3,7 , and 14 following surgery.

\section{Observation of pain-behavior responses}

Visceral pain in goats was assessed via monitoring behavioral responses to ileocecal ligament traction after TNBS-induced ileitis on days 3, 7, and 14. Following earlier evidence, ${ }^{14}$ we applied 2, 4, 6, 8, and $10 \mathrm{~N}$ forces on the ileocecal ligaments of the goats in our pretest. Traction forces of $2 \mathrm{~N}$ and $6 \mathrm{~N}$ provoked mild and moderate painful responses, respectively, and $8 \mathrm{~N}$ and $10 \mathrm{~N}$ forces induced severe behavioral pain responses. Therefore, we used traction forces of 2,4 , and $6 \mathrm{~N}$ in the formal experiments. The thread fixed to the ileocecal ligament was pulled using a dynamometer (Figure 1). Behavior responses to the $2 \mathrm{~N}$ traction force were observed for 6 seconds, and the traction stimulus was continuously increased to $4 \mathrm{~N}$ and $6 \mathrm{~N}$ in stages. Each stage lasted for 6 seconds. This procedure was repeated three times, with interstimulus intervals of 5 minutes between each test. A modified pain-behavior scale of $0-4^{15-17}$ was used for this study. Goat responses to traction were defined as pain-related behavioral scores (Table 1). Two observers who were blinded to the experimental conditions observed the behavior responses to traction forces. Pain-behavior responses to different traction forces were assessed three times and averaged as a single pain score.

\section{Sample collection}

Goats were fasted for 24 hours with free access to drinking water before the tissues were harvested. Six goats from each group were taken immediately after behavioral testing on days 3,7 , and 14 , and underwent the same laparotomy described earlier for a partial intestinal resection for collection of the
A

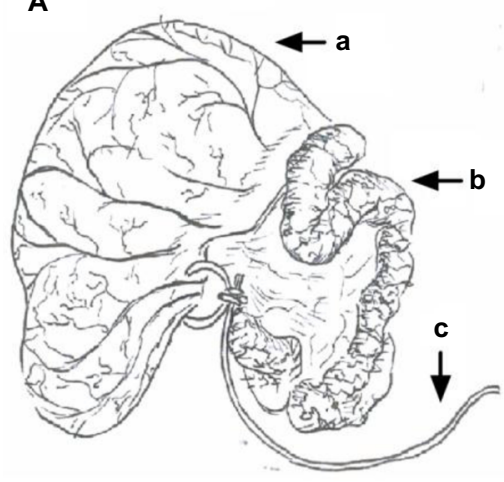

B

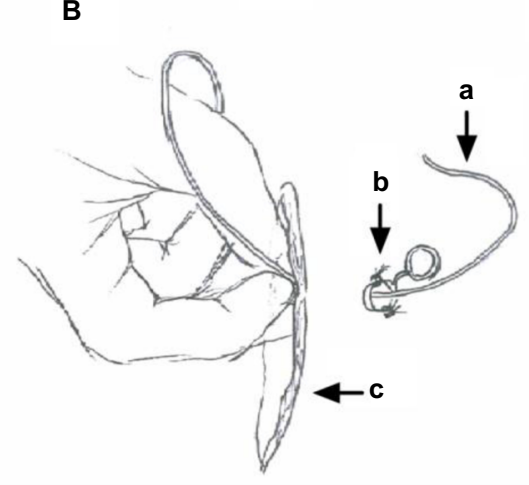

C

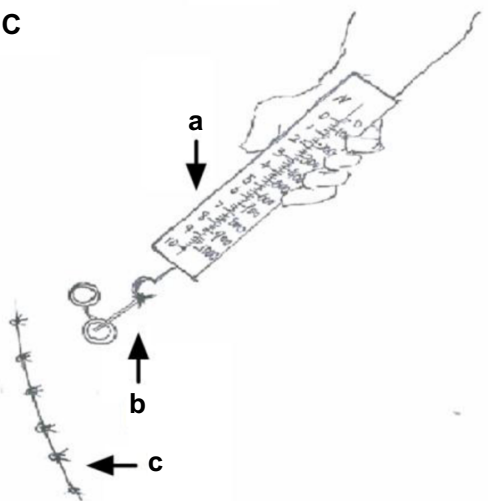

Figure I Procedure diagram.

Notes: (A) lleum and cecum exteriorized after abdominal opening: cecum (a), ileum (b), one side of the thread was tied loosely to the ileocecal ligament (c). (B) The other end of the thread was passed through an Eppendorf tube (tube fixed in the abdominal wall): thread being passed through the tube (a), tube fixed in the abdominal wall (b), flank incision (c). (C) Fixed thread on ileocecal ligament was pulled with different forces using a dynamometer: dynamometer (a), thread was used for traction (b), flank incision sutured (c). 
Table I Criteria for behavioral pain-response scores (0-4)

\begin{tabular}{lll}
\hline Scoring & Level & Description \\
\hline 0 & $\begin{array}{l}\text { Normal behavior } \\
\text { Slightly modified } \\
\text { behavior } \\
\text { Mild behavior }\end{array}$ & $\begin{array}{l}\text { No signs of discomfort } \\
\text { Rapid and shallow breathing, increased } \\
\text { heart rate, immobility of hind limbs }\end{array}$ \\
& $\begin{array}{l}\text { Restlessness, alertness, changed posture, } \\
\text { tail wagging, head back to the abdomen }\end{array}$ \\
3 & Moderate behavior & $\begin{array}{l}\text { Tail wagging, head back to the abdomen, } \\
\text { the back arching together with weight } \\
\text { shifting, occasional grunting, and teeth } \\
\text { grinding } \\
\text { Head back to the abdomen, stomping } \\
\text { feet or kicking the abdomen, repetitive } \\
\text { back arching together with weight } \\
\text { shifting, frequent grunting, and teeth } \\
\text { grinding }\end{array}$ \\
& Severe behavior \\
&
\end{tabular}

ileal tissue samples. Briefly, the ileal mesenteric arteries and veins supplying the injected region were ligated with 3-0 chromic catgut after laparotomy. Four intestinal clamps were applied on both sides of the injected part to prevent content leakage. The $6 \mathrm{~cm}$ terminal ileum of each goat was excised from $15 \mathrm{~cm}$ proximal to the ileocecal junction. The ileum segment was flushed with phosphate-buffered saline, cut longitudinally, and placed flat on a clean drape sheet. Macroscopic changes in the mucosa were observed. A $2 \times 2 \mathrm{~cm}$ block of tissue was taken from the approximate part opposite the mesentery and stored in $4 \%$ buffered formaldehyde for histopathological analyses. Another $2 \times 2 \mathrm{~cm}$ block tissue was taken from the distal part opposite the mesentery, weighed, frozen in liquid nitrogen, and transferred for storage at $-80^{\circ} \mathrm{C}$ to measure IL-1 $\beta$, IL-6, TNF $\alpha$, and myeloperoxidase (MPO) concentrations. All procedures were completed quickly.

An expert surgical team performed enteric end-to-end anastomosis immediately after the removal of ileal tissue samples, and the abdomen incision was closed as usual. The animals were housed individually in a quiet environment and monitored until full recovery from the anesthesia. Tramadol hydrochloride ( $5 \mathrm{mg} / \mathrm{kg} \mathrm{IM}$ ) was injected for 5 days for postoperative pain management. Goats received ampicillin injections $(10 \mathrm{mg} /$ $\mathrm{kg}$ IM twice daily) for up to 7 days to prevent infection. The wound was dressed with $1 \%$ povidone iodine daily until it was completely healed. The postoperative condition of the animals was monitored at least twice daily. The animals were sent to goat farms to resume their normal lives after 2 weeks of recovery.

\section{Macroscopic and microscopic observations of ileal tissue}

Macroscopic lesions were graded on days 3, 7, and 14 using a standard scale of $0-10$, where 0 is normal and 10 is maximally affected. Macroscopic scores were assessed considering ileal strictures, adhesions to surrounding tissues, ulcerations, and wall thickening, as previously described. ${ }^{18}$ Two independent observers who were unaware of the treatment designation observed all ileal samples, and their macroscopic score results were averaged. The ileal tissue samples were processed for histological evaluations using routine techniques after macroscopic assessments. Briefly, tissue segments were rinsed in saline and fixed overnight in 10\% paraformaldehyde. The fixed tissues were embedded longitudinally in paraffin and cut into $5 \mu \mathrm{m}$ sections. A total of six series of tissue sections ( $5 \mu \mathrm{m}$ thick) were obtained. Three sections were stained with hematoxylin and eosin, and the other three sections were stained with $0.5 \%$ toluidine blue. Two investigators were blindly assigned to examine and score all sections according to inflammation degree, lesion depth, hyperemia/edema, and inflammatory cell infiltration, as previously described. ${ }^{19}$ The slides were examined and photographed to characterize the histopathological changes using light microscopy (Eclipse 80I; Nikon Corporation, Tokyo, Japan) with a $10 \times$ objective lens. The number of mast cells per microscopic view $\left(0.16 \mathrm{~mm}^{2}\right.$ at $\times 200$ magnification) was counted in three areas per slice and averaged. Representative photomicrographs were obtained at $20 \times$ magnifications.

\section{Measurement of cytokines and myeloperoxidase}

Ileal tissue was ground and homogenized in $1 \mathrm{~mL}$ phosphatebuffered saline, $\mathrm{pH} 7.2$, at $4^{\circ} \mathrm{C}$ containing PMSF protein inhibitor. Homogenates were shaken at $60 \mathrm{~Hz}$ for 90 seconds, and the solution was centrifuged at 5,000 $g$ at $4{ }^{\circ} \mathrm{C}$ for 15 minutes. Protein concentrations were determined using a NanoDrop Spectrophotometer (Thermo Fisher Scientific, Waltham, MA, USA). MPO concentration was measured using an enzyme-linked immunosorbent assay (ELISA) kit (eBioscience Inc, San Diego, CA, USA) according to the manufacturer's protocol. IL-1 $\beta$, IL-6, and TNF $\alpha$ were measured using ELISA kits (NeoBioscience, Shenzhen, PRC) following the manufacturer's instructions. Each sample was analyzed in triplicate, and values are presented as $\mathrm{pg} / \mathrm{mg}$.

\section{Statistical analysis}

Data are presented as mean \pm standard deviation, and all statistical calculations were performed using SPSS 17.0 statistical software (SPSS Inc, Chicago, IL, USA). One-way analysis of variance followed by Student's $t$-test was used to compare the parametric values (body weight, TNF $\alpha$, IL-1 $\beta$, IL-6, and MPO concentrations) of the TNBS- and saline-treated goats. Nonparametric values (macroscopic and microscopic scores, mast cells, and behavior scores) were analyzed using Kruskal-Wallis 
analysis of variance followed by Mann-Whitney tests. The correlation between traction forces and behavioral responses was assessed by computing Spearman's correlation coefficient. $P$-values less than 0.05 were considered statistically significant in all statistical analyses.

\section{Results}

\section{Behavioral responses to traction on ileocecal ligament}

Graded traction $(2,4$, and $6 \mathrm{~N})$ on the ileocecal ligaments of goats resulted in pain responses on days 3,7 , and 14 . The experimental goats exhibited significant signs, such as restlessness, rapid breathing, guarding, tail wagging, curling of the lips, neck movement, and changes in posture, when traction was applied. The TNBS-treated goats exhibited significantly increased behavioral responses to ileocecal stimuli on day 3 with traction of $2 \mathrm{~N}(P=0.007), 4 \mathrm{~N}(P=0.005)$, and $6 \mathrm{~N}(P=0.009)$, on day 7 with traction of $2 \mathrm{~N}(P=0.039)$,
$4 \mathrm{~N}$, and $6 \mathrm{~N}(P=0.006)$, and on day 14 with traction of 2 $\mathrm{N}(P=0.252), 4 \mathrm{~N}(P=0.024)$, and $6 \mathrm{~N}(P=0.007)$ compared to the saline-treated goats (Figure $2 \mathrm{~A}-\mathrm{C}$ ). Pain responses positively correlated $\left(R^{2}=0.620, P=0.000\right)$ to traction on the ileocecal ligament with 2, 4, and $6 \mathrm{~N}$ (Figure 2D).

\section{Body-weight changes in treated goats}

Saline-treated goats exhibited transient body-weight loss of $2.06 \pm 2.97 \mathrm{~kg}$ on day 3 , which gradually increased to $2.75 \pm 1.91$ and $9.09 \pm 4.61 \mathrm{~kg}$ on days 7 and 14, respectively. Body weights in the TNBS-treated goats decreased markedly compared to the saline-group goats, and this group developed diarrhea on days 3 and 7. The TNBS-treated goats lost 3.22 $\pm 1.7,2.89 \pm 2.01$, and $3.19 \pm 1.83 \mathrm{~kg}$ on days 3,7 , and 14 , respectively. However, the body weight of the TNBS group was not significantly different $(P=0.61)$ from the saline group on day 3. Significant differences were observed between the groups on days 7 and 14 ( $P=0.004$ and $P=0.016$, respectively) (Figure 3C).
A

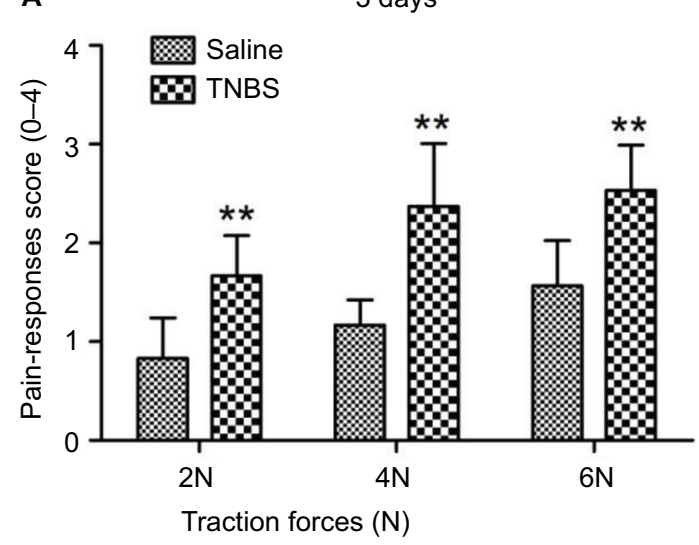

C

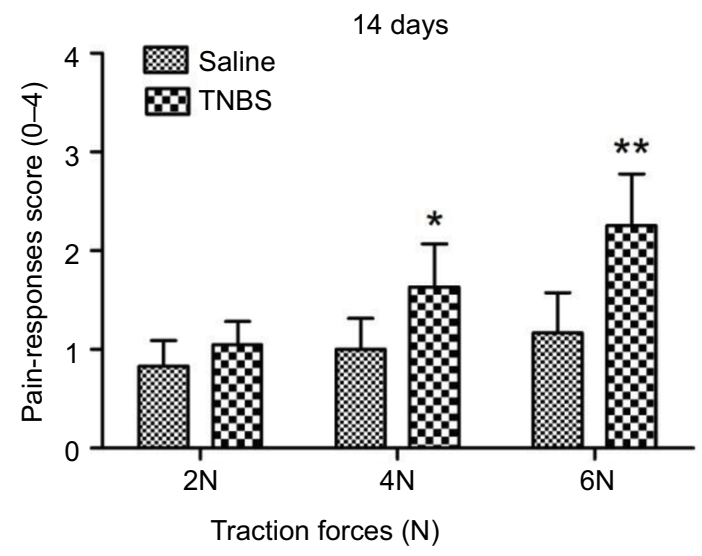

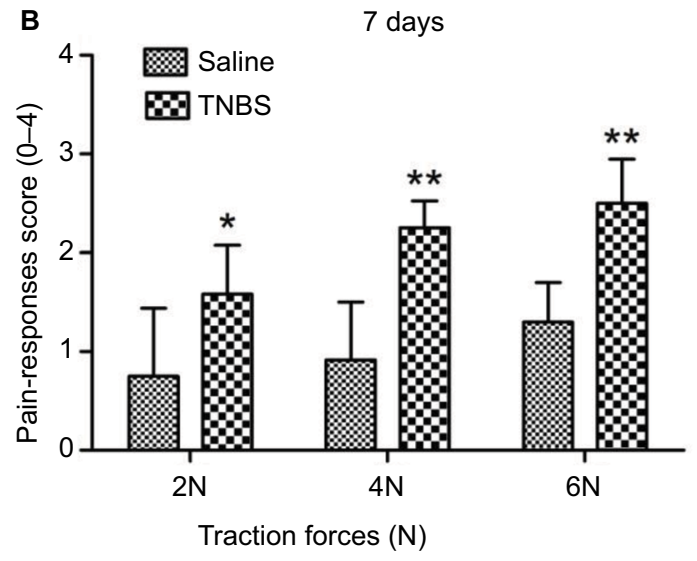

D

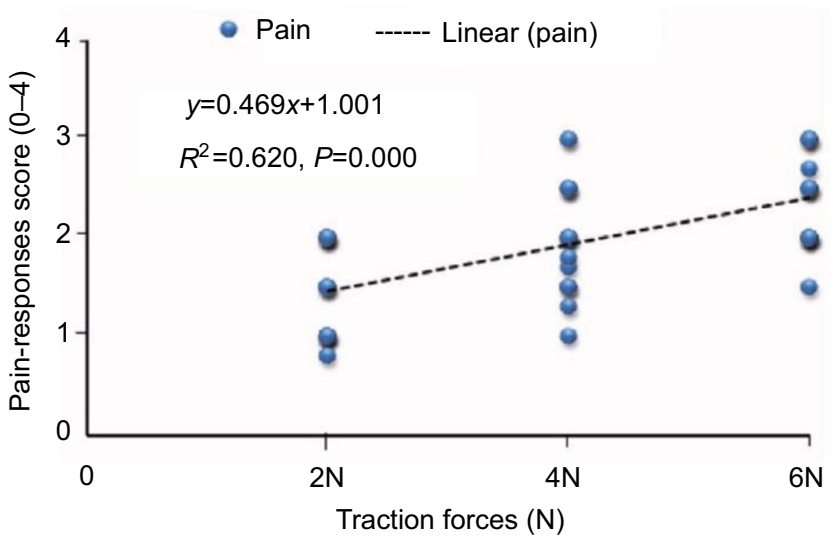

Figure 2 Pain-behavior responses to traction on the ileocecal ligament after 2,4,6-trinitrobenzenesulfonic acid (TNBS) treatment in goats.

Notes: (A-C) Bars indicate pain behavior-response scores between TNBS-treated goats and saline-treated goats on days 3, 7, and I4. Graphs represent mean \pm standard deviation ( $\mathrm{n}=6$ goats). $* P<0.05$, $* * P<0.0$ I compared to the saline group (Kruskal-Wallis analysis of variance followed by Mann-Whitney test). (D) Correlation between different traction forces $(2,4$, and $6 \mathrm{~N})$ and pain-response scores (Spearman's correlation test). 
A
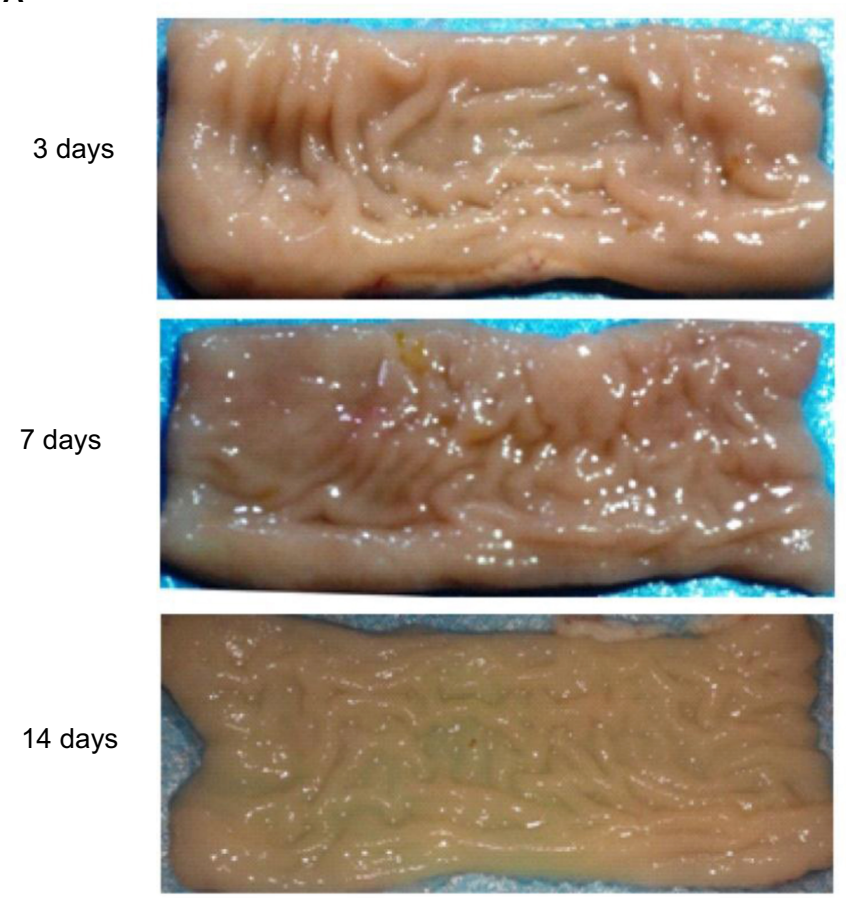

TNBS
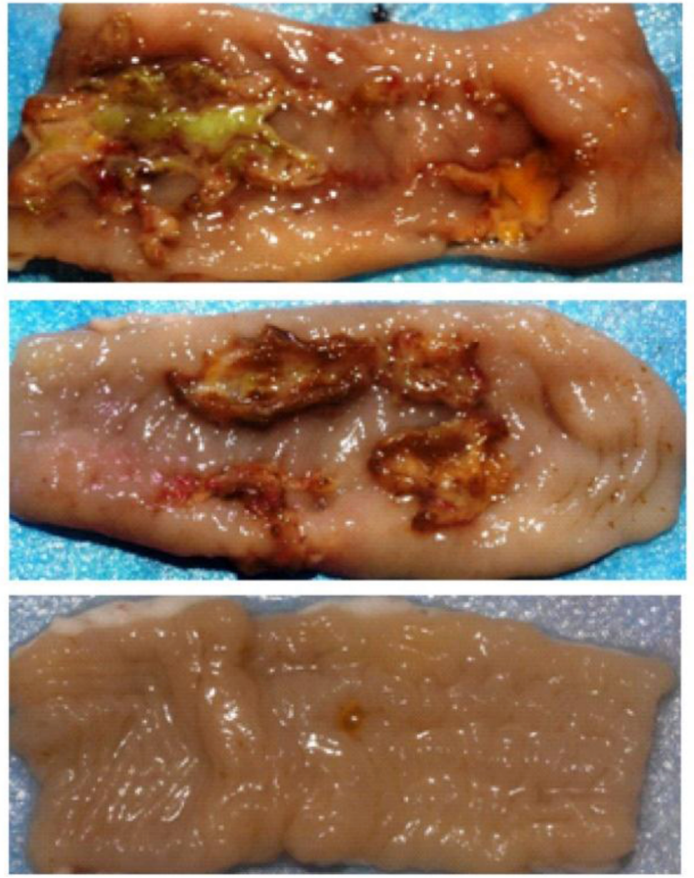

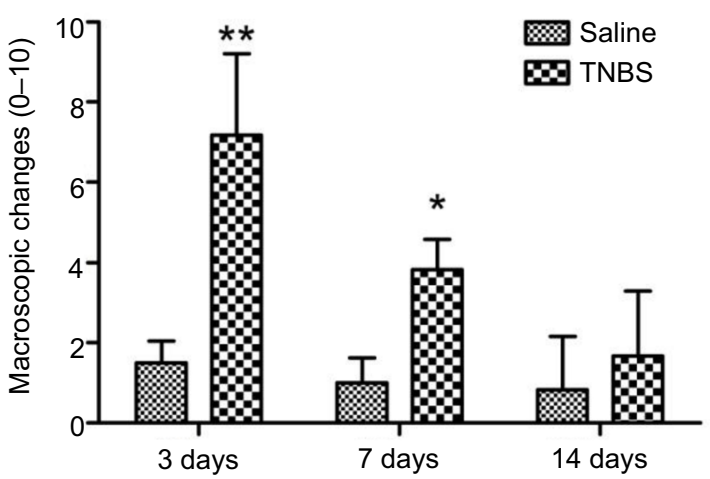

C

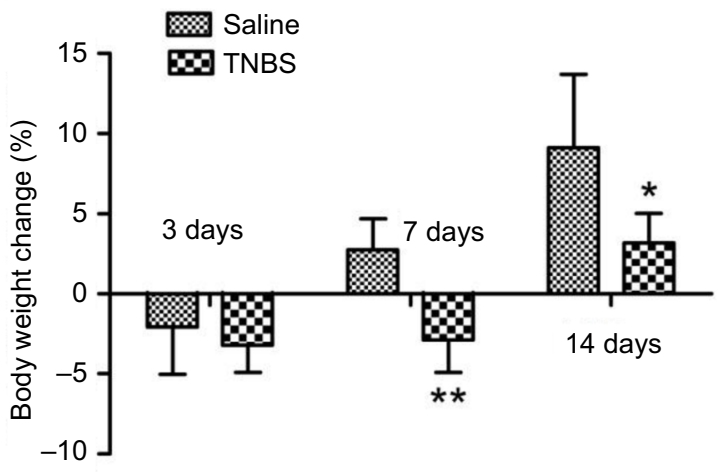

Figure 3 Effects of 2,4,6-trinitrobenzenesulfonic acid (TNBS) administration on ileal macroscopic changes and body weight in goats.

Notes: (A) Saline-treated ileum appeared quite intact on days 3, 7, and I4; administration of $1.2 \mathrm{~mL}$ TNBS (30 mg TNBS in $30 \%$ of ethanol) into the ileal wall produced mucosal erosions, necrosis, extensive ulceration, hemorrhage, and adhesions on days 3 and 7. (B) Macroscopic damage scores in TNBS-treated goats increased on days 3 and $7(P=0.003)$ compared to saline-treated goats. Graphs represent mean \pm standard deviation ( $n=6$ samples). ${ }^{*} P<0.05$, $* * P<0.01$ compared to the saline group (Kruskal-Wallis followed by Mann-Whitney $U$ test). (C) Decreased percentage body weight in TNBS-treated goats on day $3(P=0.61)$, day $7(P=0.004)$, and day I4 ( $P=0.016)($ one-way analysis of variance followed by Student's $t$-test).

\section{Ileal macroscopic features of treated goats}

Macroscopic examinations of ilea revealed no apparent changes $(P>0.05)$ in the saline-treated goats during the experiment. The TNBS-treated ileal tissue revealed mucosal erosions, necrosis, extensive ulceration, hemorrhage, and adhesions on days 3 and 7, but did not show any obvious signs of inflammation except wall thickening on day 14 (Figure 3A). No apparent changes were observed in adjacent visceral organs, such as the jejunum, cecum, colon, and rectum. Changes in macroscopic scores in the TNBS-treated ilea were 7.16 \pm 2.04 and $3.83 \pm 0.75$ on days 3 and 7 , respectively, which were higher $(P=0.003)$ than those of the saline-treated ilea $(1.5 \pm 0.54$ and $1 \pm 0.63$, respectively) (Figure $3 \mathrm{~B})$. There was no significant difference $(P=0.303)$ between the groups on day 14 .

\section{Ileal microscopic changes in experimental goats}

No apparent pathological changes were found in salineinjected ilea during the experiment (Figure 4A). The TNBStreated ilea exhibited severe inflammatory changes, with shallow crypt architecture, inflammatory cell infiltration, 

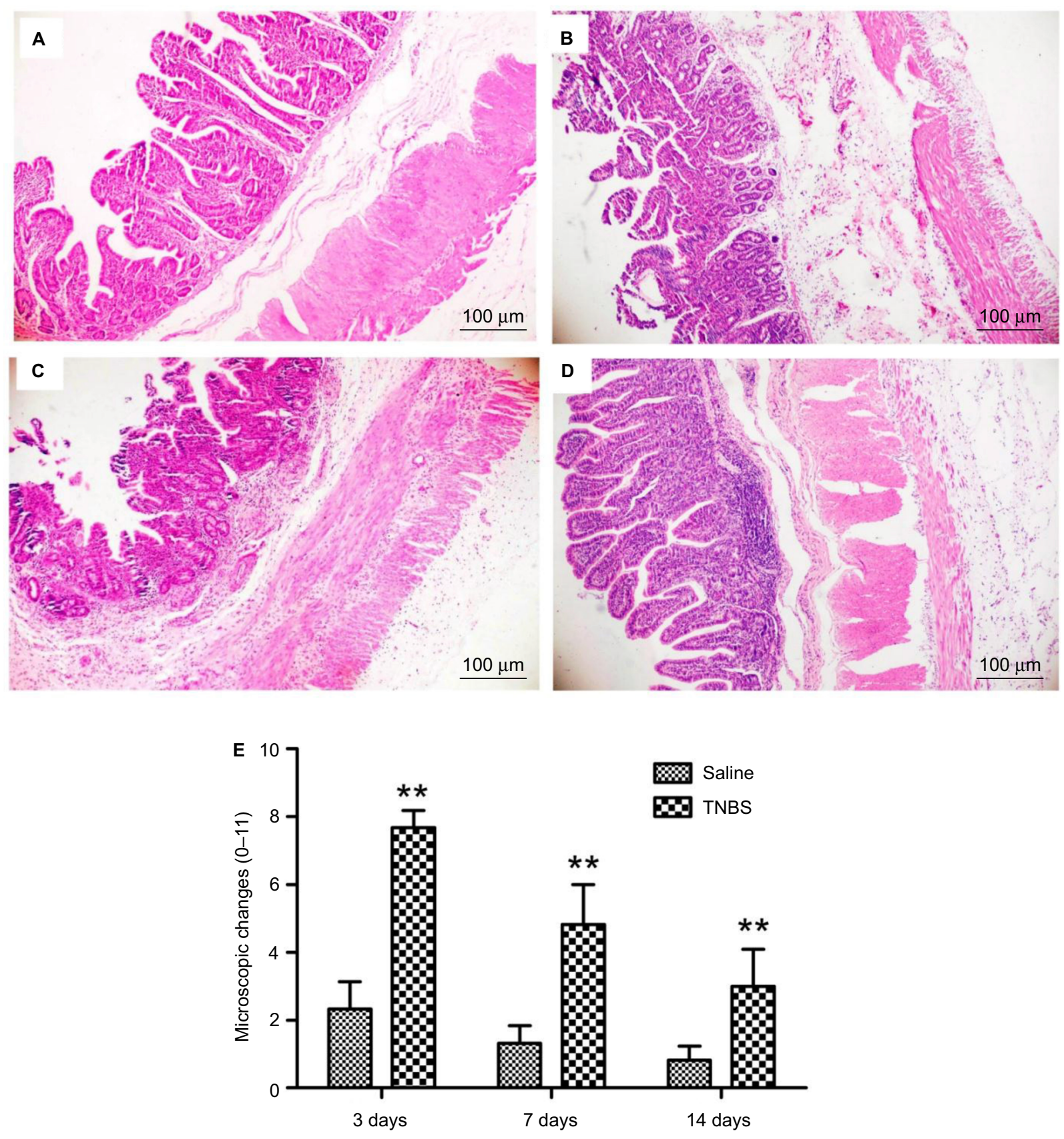

Figure 4 Effects of 2,4,6-trinitrobenzenesulfonic acid (TNBS) administration on histopathology of the ileum in goats.

Notes: Microscopic changes of the ileum stained with hematoxylin and eosin (10x magnification). (A) Saline-treated ilea of goats show intact epithelium, absence of edema, and intact muscle architecture. (B, C) After administration of $1.2 \mathrm{~mL}$ of a TNBS solution (30 mg TNBS in $30 \%$ of ethanol) into the ileum wall, the ileum exhibited severe shallow crypt architecture, inflammatory cell infiltration, and a thickened wall due to edema and hyperemia on days 3 and 7 . (D) TNBS-treated goats exhibited low-grade ileal inflammation on day 14. (E) Microscopic change scores increased in TNBS-treated goats on day $3(P=0.000)$, day $7(P=0.000)$, and day $14(P=0.005)$ compared to saline-treated goats. Graph represents mean \pm standard deviation ( $n=6$ samples). $* * P<0.01$ compared to the saline group (Kruskal-Wallis analysis of variance followed by Mann-Whitney test).

and thickened walls due to edema and hyperemia on days 3 and 7 (Figure 4B and C). At day 14, microscopic changes in the TNBS group appeared as low-grade inflammation in ileal tissue (Figure 4D). The changes in microscopic scores in TNBS-treated ilea were $7.66 \pm 0.51,4.83 \pm 1.16$, and $3 \pm 1.09$ on days 3,7 , and 14 , respectively, which were higher $(P=0.003)$ than the $2.33 \pm 0.81,1.33 \pm 0.51$ and $0.83 \pm 0.4$ score changes in the saline control group (Figure 4E). The numbers of mast cells in the ileal tissue of the saline-treated goats were $21.37 \pm 23.15,21.95 \pm 6.06$, and $20.87 \pm 16.35$ on days 3,7 , and 14, respectively (Figure 5A). Compared with the saline group, the number of mast cells in the TNBS goats was higher on days 3 and 7 (55.12 $\pm 11.62, P=0.019$ and $48.37 \pm 15.63$, $P=0.004$; Figure 5B). However, there was no difference $(P=0.257)$ in mucosal mast cells between the groups on day 14 (Figure 5C). 

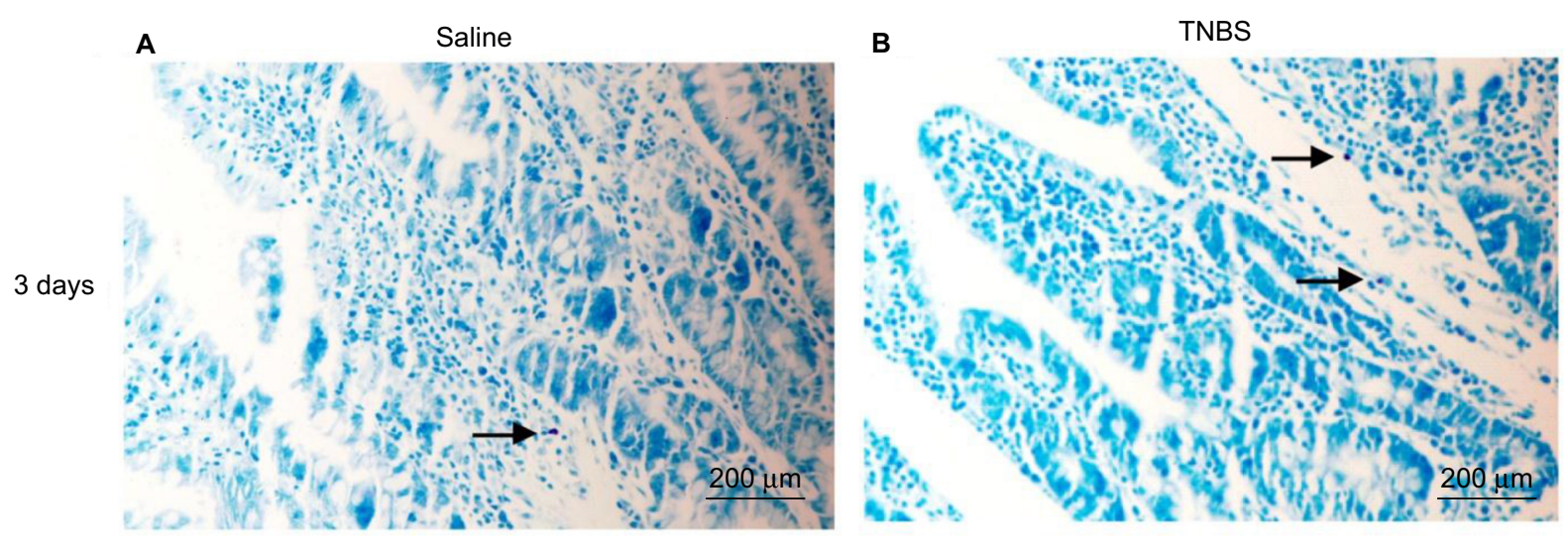

C

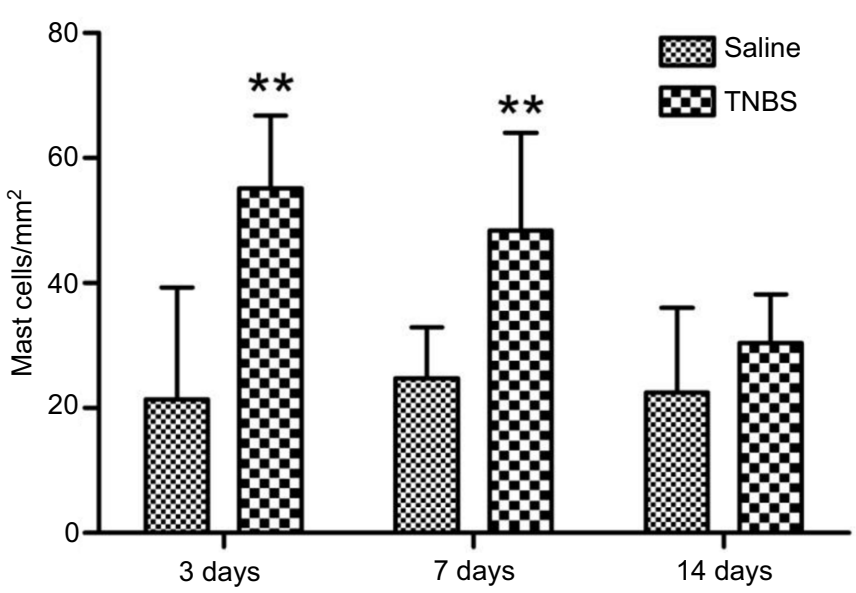

Figure 5 Effects of 2,4,6-trinitrobenzenesulfonic acid (TNBS) administration on mast-cell counts.

Notes: llea of goats 3, 7, and 14 days after treatment (20x magnification, stained using toluidine blue). Arrows show the mast cells in (A) Saline treated group and (B) TNBS treated group. (C) Mast cell counts in ilea of TNBS-treated goats and saline-treated goats were $55.12 \pm I I .62$ ( $P=0.019)$ and $48.37 \pm 15.63$ ( $P=0.004)$ on days 3 and 7 . Graphs represent mean \pm standard deviation ( $n=6$ samples). $* * P<0.01$ compared to the saline group. (Kruskal-Wallis analysis of variance followed by Mann-Whitney test).

\section{Changes in ileal cytokines and myeloperoxidase concentrations}

No changes were observed in the concentrations of IL-1 $\beta$, IL-6, or TNF $\alpha$ in the saline-treated group during the experiment. The concentrations of IL-1 $\beta$ and IL-6 in TNBS-treated goats were 41.7 $\pm 7.89(P=0.000)$ and $30.66 \pm 9.68(P=0.000)$ on day 3 , $19.78 \pm 4.82(P=0.006)$ and $19.86 \pm 2.89(P=0.000)$ on day 7 , and $13.16 \pm 2.12(P=0.026)$ and $14.44 \pm 3.06(P=0.035)$ on day 14 , respectively, which were higher than those of the saline-treated goats (Figure 6A and B). The TNF $\alpha$ levels in the TNBS-treated ilea were $27.1 \pm 5.65$ and $21.14 \pm 4.02$ on days 3 and 7 , respectively, which were higher $(P=0.003$ and $P=0.045)$ than those of the saline-treated ilea (15.8 \pm 4.03 and $16.42 \pm 3.05$, respectively) (Figure 6C). MPO concentrations in the TNBS-treated ileum tissue were $2,084.16 \pm 433.52$ and $1,537.35 \pm 354.38$ on days 3 and 7, respectively, which were higher $(P=0.000$ and $P=0.006)$ than those of the saline group ( $975.4 \pm 147.5$ and $938.28 \pm 219.71$, respectively) (Figure 6D). No differences in TNF $\alpha$ or MPO concentrations were found between the groups on day 14 .

\section{Discussion}

Visceral pain is primarily the consequence of the stretching of the viscera and injury or inflammation to the gastrointestinal tract. The mechanisms of visceral pain are not well studied compared to somatic pain. Visceral pain is distinctly different from somatic nociception in its clinical, physiological, and in particular neurological mechanisms..$^{20}$ Primary visceral afferents are found in the serosa, muscle, and mucosa of the gastrointestinal tract, which may be sensitized and contribute to disproportionate pain states in response to injury or inflammation via cytokines and protease release. ${ }^{21,22}$ Visceral pain is more difficult to assess, because it tends to be diffuse in nature and occur intermittently as a result of complex processes involving peripheral and central sensitization. ${ }^{6,23}$ Visceral pain is the result of multiple factors, including stimuli (mechanical versus chemical), etiology (inflammatory versus postinflammatory) and time course (acute versus chronic). There are numerous chemically induced inflammation experimental studies in the literature that indicate the potential benefit of 

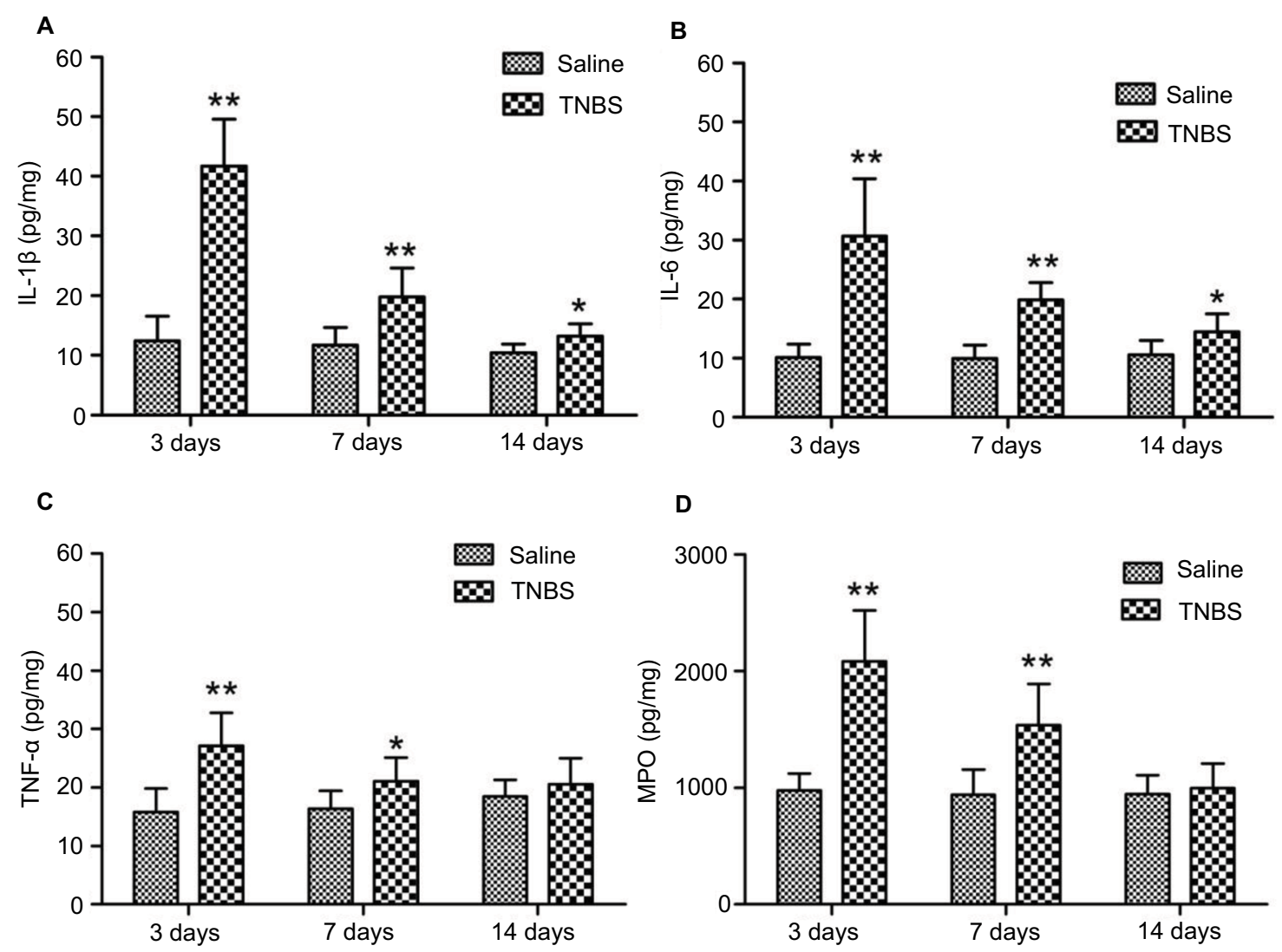

Figure 6 Effects of 2,4,6-trinitrobenzenesulfonic acid (TNBS) administration on cytokine (IL-I $\beta$, IL-6, TNF $\alpha$ ) and myeloperoxidase (MPO) concentrations in ilea of goats. Notes: Quantified using enzyme-linked immunosorbent assay on days 3, 7, and I4. (A, B). At days 3, 7, and I4, concentrations of IL-I $\beta$ and IL-6 were significantly increased ( $P=0.000,0.006, P=0.026$ and $P=0, P=0, P=0.035$, respectively) in TNBS-treated goats compared to saline-treated goats. (C, D) Concentrations of TNF $\alpha$ and MPO were significantly increased on day $3(P=0.003$ and $P=0.045$, respectively) and day 7 ( $P=0.000$ and $P=0.006)$ in TNBS-treated goats compared to saline-treated goats. Data presented as mean \pm standard deviation, $n=6 ; * P<0.05$, $* * P<0.0$, compared to the saline group (one-way analysis of variance followed by Student's $t$-test).

designing an appropriate animal model of pain originating from the small intestine to evaluate the efficacy of treatments for visceral pain and thereby improve our understanding of the underlying mechanisms.

Experimental models of TNBS-induced intestinal inflammation are widely used for pathological and pharmacological studies. TNBS-induced colitis in rats was originally described as a model for Crohn's disease (CD) and ulcerative colitis. ${ }^{24}$ TNBS was administered into the ileal lumen to induce ileitis in several studies. The inflammatory responses of the ileum are different from the well-characterized response of the colon in severity and duration. The doses and concentrations of TNBS differ between studies, the methodologies are inconsistent, and no standardized protocol exists. Moreels et al injected $85 \mathrm{mg} / \mathrm{kg}$ TNBS in $40 \%$ ethanol into rat ilea and found a marked inflammatory reaction based on MPO concentration and histopathological changes at 36 hours that lasted up to 7 days. ${ }^{25}$ TNBS was administered $(30 \mathrm{mg} / \mathrm{kg}$ in $30 \%$ ethanol) in the ilea of guinea pigs, and both clinical and histopathological evidence of ileitis was observed for up to 7 days. ${ }^{26}$ Merritt et $\mathrm{al}^{27}$ induced porcine ileitis via administration of TNBS (500 or 1,000 $\mathrm{mg}$ in 33 to $75 \%$ ethanol), and confirmed inflammation up to 7 days. All of these studies demonstrated a short duration of ileitis induced by TNBS. The characteristics of visceral pain (such as dynamic change) were not clear in these reports, because it was not assessed. Other methods have been used to induce ileitis: 4\% paraformaldehyde was injected at multiple sites into the ileal wall of pigs, and apparent ileitis was observed at 3 days. $^{28}$ This method exhibited good reproducibility compared with intramural injections, and required a lower chemical dose. Ileitis induced by TNBS injection into the ileal wall has not been reported previously.

The present study injected TNBS (30 mg dissolved in $30 \%$ ethanol) into the ileal wall, which increased the macroscopic change scores, mast-cell numbers, and MPO and TNF $\alpha$ concentrations on days 3 and 7 and increased the microscopic change scores and IL-1 $\beta$ and IL-6 levels on days 3 and 14. Macroscopic and microscopic change scores and MPO and cytokine concentrations revealed that TNBS-induced ileitis exhibited maximum severity on day 3 and then declined gradually, but remained significantly 
higher on day 14. Inflammatory severity was influenced by the contact time of TNBS with the ileal mucosa, due to intestine emptying and peristalsis. ${ }^{27}$ The remarkable inflammatory lesions and good reproducibility observed in our experiment suggest that a lower dose of TNBS injected directly into the ileum wall facilitated its full action on the intestinal tissue. Previous studies used graded traction forces to evaluate the efficacy of analgesics and inhalational anesthetics in animal models. ${ }^{13,14}$ However, these studies were performed for 1 or 5 hours in the anesthetized animals. The present study used graded traction with 2, 4, and $6 \mathrm{~N}$ on the ileocecal ligament, which correlated positively with pain-behavior responses on days 3-14. Abundant evidence suggests that the activation of various inflammatory cells (mast cells, macrophages, neutrophils, and T lymphocytes) releases inflammatory mediators, such as cytokines (IL$1 \beta$, IL-6, TNF $\alpha$ ), which contribute to the sensitization of nociceptors and exacerbate pain ${ }^{17,29-31}$ under inflammatory conditions. Sustained depolarization of the nerve terminals by inflammatory mediators would prolong firing and result in increased afferent traffic to the central nervous system. These changes ultimately lead to an increased perception of visceral pain.

Visceral pain originating from the small intestine is commonly seen in clinical practice. Visceral hyperalgesia is highly prevalent in most bowel diseases, and it is a key pathophysiological mechanism involved in pain perception. ${ }^{21,32,33}$ Rectal balloon distention is generally used to measure visceral hyperalgesia. A previous study ${ }^{34}$ reported that patients with gastrointestinal disorders during acute inflammatory changes exhibited enhanced visceral sensitivity when rectal distention was applied. Faure and Giguère ${ }^{35}$ observed that children and adolescents with CD exhibited visceral hypersensitivity and anxiety to rectal distension. A study conducted in patients with $\mathrm{CD}$ demonstrated decreased or normal sensitivity to rectal distension. ${ }^{36}$ Bernstein et $\mathrm{al}^{37}$ compared rectal afferent mechanisms in $\mathrm{CD}$ patients with diarrheapredominant inflammatory bowel-syndrome patients. CD patients exhibited reduced pain, and patients with inflammatory bowel syndrome had higher pain responses during rectal distension. A recent study reported the evidence of visceral hyperalgesia in TNBS-induced ileitis rats. ${ }^{38}$ This discrepancy may have been due to region-specific degrees of gastrointestinal tract inflammation and pain sensation. Pain-signal transmission in patients with small-intestine diseases occurs via thoracosplanchnic pathways. The decreased hyperalgesia observed in $\mathrm{CD}$ patients may result from signal interactions at the spinal cord level and via descending bulbospinal inhibition of sacral dorsal horn neurons in response to rectal distension. ${ }^{37}$ The present study used different traction forces on the ileocecal ligament, which shifted to the inflammatory ileum and provoked visceral pain. This response to traction resulted from mesenteric and ileal pain, which is consistent with pathological situations.

\section{Conclusion}

This study is the first experimental study to investigate visceral pain by using ileocecal ligament traction on an inflamed ileum. TNBS injection (30 mg in 30\% ethanol) into the ileal wall of goats produced apparent transmural inflammatory lesions accompanied by significant pain-behavior scores to graded traction $(2,4$, and $6 \mathrm{~N})$ on the ileocecal ligament on days 3-14. Traction on the ileocecal ligament was a reliable and repeatable means to produce pain-behavior responses for scaling visceral pain intensity. This model may be convenient for the further exploration of the mechanisms underlying visceral pain and for evaluating the efficacy of analgesics and other new therapeutic regimens for the proper treatment of gastrointestinal disorders.

\section{Acknowledgments}

The authors thank Wang Shao Wei, College of Veterinary Medicine for his expert technical assistance during the experiments. This study was supported by a fund from the National Natural Science Foundation of China (31472246).

\section{Disclosure}

The authors report no conflicts of interest in this work.

\section{References}

1. Dilauro S, Crum-Cianflone NF. Ileitis: when it is not Crohn's disease. Curr Gastroenterol Rep. 2010;12(4):249-258.

2. Davis MP. Drug management of visceral pain: concepts from basic research. Pain Res Treat. 2012;2012:265605.

3. Zakka TM, Teixeira MJ, Yeng LT. Abdominal visceral pain: clinical aspects. Rev Dor. 2013;14(4):311-314.

4. Bueno L, Fioramonti J, Delvaux M, Frexinos J. Mediators and pharmacology of visceral sensitivity: from basic to clinical investigations. Gastroenterology. 1997;112(5):1714-1743.

5. Greenwood-Van Meerveld B, Prusator DK, Johnson AC. Animal models of gastrointestinal and liver diseases: animal models of visceral pain pathophysiology, translational relevance, and challenges. Am J Physiol Gastrointest Liver Physiol. 2015;308(11):G885-G903.

6. Sengupta JN. Visceral pain: the neurophysiological mechanism. Handb Exp Pharmacol. 2009;(194):31-74.

7. Chen DH, Chen HR, Kasim A, Liu ZQ, Zheng PY. Probiotics alleviates intestinal immune dysfunction in a mouse model of irritable bowel syndrome. Life Sci J. 2014;11(6):309-316.

8. Gawade SP. Acetic acid induced painful endogenous infliction in writhing test on mice. J Pharmacol Pharmacother. 2012;3(4):348.

9. Saha S, Guria T, Singha T, Maity TK. Evaluation of analgesic and antiinflammatory activity of chloroform and methanol extracts of Centella asiatica Linn. ISRN Pharmacol. 2013;2013:789613. 
10. Adam B, Liebregts T, Gschossmann JM, et al. Severity of mucosal inflammation as a predictor for alterations of visceral sensory function in a rat model. Pain. 2006;123(1-2):179-186.

11. Yang J, Yao M, Jiang X, Wang L. Establishment of model of visceral pain due to colorectal distension and its behavioral assessment in rats. World J Gastroenterol. 2006;12(17):2781-2784.

12. Feng B, La JH, Tanaka T, Schwartz ES, McMurray TP, Gebhart GF. Altered colorectal afferent function associated with TNBS-induced visceral hypersensitivity in mice. Am J Physiol Gastrointest Liver Physiol. 2012;303(7):G817-G824.

13. Holzer-Petsche U, Brodacz B. Traction on the mesentery as a model of visceral nociception. Pain. 1999;80(1-2):319-328.

14. Boscan P, Monnet E, Mama K, et al. A dog model to study ovary, ovarian ligament and visceral pain. Vet Anaesth Analg. 2011;38(3):260-266.

15. Molony V, Kent JE. Assessment of acute pain in farm animals using behavioral and physiological measurements. J Anim Sci. 1997;75(1):266-272.

16. Stasiak KL, Maul D, French E, Hellyer PW, Van de Woude S. Speciesspecific assessment of pain in laboratory animals. Contemp Top Lab Anim Sci. 2003;42(4):13-20.

17. Kirscher F, Bruckmaier RM, Spadavecchia C, et al. Quantitative assessment of physiological and behavioural parameters in healthy dairy cows evoked by transcutaneous electrical nerve stimulation of the udder. Vet J. 2012;192(2):183-188.

18. Mourelle M, Guarner F, Malagelada JR. Polyunsaturated phosphatidylcholine prevents stricture formation in a rat model of colitis. Gastroenterology. 1996;110(4):1093-1097.

19. Engel MA, Kellermann CA, Rau T, Burnat G, Hahn EG, Konturek PC. Ulcerative colitis in AKR mice is attenuated by intraperitoneally administered anandamide. J Physiol Pharmacol. 2008;59(4): 673-689.

20. Cervero F, Laird JM. Visceral pain. Lancet. 1999;353(9170):2145-2148.

21. Bueno L, Fioramonti J. Visceral perception: inflammatory and noninflammatory mediators. Gut. 2002;51 Suppl 1:i19-i23.

22. Zhang JM, An J. Cytokines, inflammation, and pain. Int Anesthesiol Clin. 2007;45(2):27-37.

23. Mayer EA, Gebhart GF. Basic and clinical aspects of visceral hyperalgesia. Gastroenterology. 1994;107(1):271-293.

24. Morris GP, Beck PL, Herridge MS, Depew WT, Szewczuk MR, Wallace JL. Hapten-induced model of chronic inflammation and ulceration in the rat colon. Gastroenterology. 1989;96(3):795-803.
25. Moreels TG, De Man JG, Dick JM, et al. Effect of TNBS-induced morphological changes on pharmacological contractility of the rat ileum. Eur J Pharmacol. 2001;423(2-3):211-222.

26. Nurgali K, Qu Z, Hunne B, Thacker M, Pontell L, Furness JB. Morphological and functional changes in guinea-pig neurons projecting to the ileal mucosa at early stages after inflammatory damage. $J$ Physiol. 2011;589(Pt 2):325-339.

27. Merritt AM, Buergelt CD, Sanchez LC. Porcine ileitis model induced by TNBS-ethanol instillation. Dig Dis Sci. 2002;47(4):879-885.

28. Czaja K, Kaleczyc J, Sienkiewicz W, Lakomy M. The influence of experimental ileitis on the neuropeptide coding of enteric neurons in the pig. Pol J Vet Sci. 2005;8(2):155-163.

29. Metcalfe DD, Baram D, Mekori YA. Mast cells. Physiol Rev. 1997; 77(4):1033-1079.

30. Bueno L, Fioramonti J, Garcia-Villar R. Pathobiology of visceral pain: molecular mechanisms and therapeutic implications. III. Visceral afferent pathways: a source of new therapeutic targets for abdominal pain. Am J Physiol Gastrointest Liver Physiol. 2000;278(5):G670-G676.

31. Zhang J, Shen B, Lin A. Novel strategies for inhibition of the p38 MAPK pathway. Trends Pharmacol Sci. 2007;28(6):286-295.

32. De Winter BY, Deiteren A, De Man JG. Novel nervous system mechanisms in visceral pain. Neurogastroenterol Motil. 2016;28(3):309-315.

33. Coss-Adame E, Rao SS. Brain and gut interactions in irritable bowel syndrome: new paradigms and new understandings. Curr Gastroenterol Rep. 2014;16(4):379.

34. Fuchs E, Flügge G. Modulation of binding sites for corticotropinreleasing hormone by chronic psychosocial stress. Psychoneuroendocrinology. 1995;20(1):33-51.

35. Faure C, Giguère L. Functional gastrointestinal disorders and visceral hypersensitivity in children and adolescents suffering from Crohn's disease. Inflamm Bowel Dis. 2008;14(11):1569-1574.

36. Bremner JD, Randall P, Vermetten E, et al. Magnetic resonance imagingbased measurement of hippocampal volume in posttraumatic stress disorder related to childhood physical and sexual abuse: a preliminary report. Biol Psychiatry. 1997;41(1):23-32.

37. Bernstein $\mathrm{CN}$, Niazi N, Robert $\mathrm{M}$, et al. Rectal afferent function in patients with inflammatory and functional intestinal disorders. Pain. 1996;66(2-3):151-161.

38. Shah MK, Wan J, Janyaro H, Tahir AH, Cui L, Ding MX. Visceral hypersensitivity is provoked by 2,4,6-trinitrobenzene sulfonic acidinduced ileitis in rats. Front Pharmacol. 2016;7(214):1-13.
Journal of Pain Research

\section{Publish your work in this journal}

The Journal of Pain Research is an international, peer reviewed, open access, online journal that welcomes laboratory and clinical findings in the fields of pain research and the prevention and management of pain. Original research, reviews, symposium reports, hypothesis formation and commentaries are all considered for publication.

\section{Dovepress}

The manuscript management system is completely online and includes a very quick and fair peer-review system, which is all easy to use. Visit http://www.dovepress.com/testimonials.php to read real quotes from published authors. 